\title{
GRAY SCALE IMAGE COMPRESSION BASED ON WAVElet TransForm AND LiNEAR PREDiCTION
}

\author{
Arya Devi P S ${ }^{1}$ and M.G.Mini ${ }^{2}$ \\ Department of Electronics, Model Engineering College, Kochi, India \\ 1aryaps@mec.ac.in, ${ }^{2}$ mininair@mec.ac.in
}

\begin{abstract}
Every year, several terabytes of image data-both medical and non medical-are engendered so that the requisition for image compression is substantiated. In this paper, the correlation properties of wavelets are harnessed in linear predictive coding to compress images. The image is decomposed using a one dimensional wavelet transform. The highest level wavelet transform coefficients and few detail coefficients in every level are retained. Using linear prediction on these coefficients the image is reconstructed. The prediction is done in both the dimensions, so the numbers of coefficients retained in detail subbands are less. With less predictors and samples from the original wavelet coefficients compression can be achieved. The results are appraised in objective and subjective manner with real world and medical images. The results are also verified on ModelFest database.
\end{abstract}

\section{KEYWORDS}

Image Compression, bits per pixel (bpp), wavelet transform, linear predictive coding (LPC), correlation

\section{INTRODUCTION}

Image compression is a key issue to be addressed in the area of transmission and storage of images. The storage and transmission of large volumes of image data is a challenging task owing to limited storage space and bandwidth. With the emerging technologies, there are promises of unlimited bandwidth. But the need and availability for images outgrow the increase in network capacity. The high costs involved in providing large bandwidth and huge storage space further necessitates the need for image compression. Image compression finds its application in various fields ranging from satellite imaging, medical imaging to teleconferencing, HDTV etc. Compressing an image is the process of reducing the size of the image, without degrading the image quality by exploiting its redundancy and irrelevancy. Even as many techniques available and emerging in the field of image compression, the demand for digital image transmission indicate that there is always room for better and novel methods.

Wavelet Transform (WT) is a tool that allows multi resolution analysis of an image. It can extract relevant information from an image and can adapt to human visual characteristics. WT decomposes an image into a set of different resolution sub-images corresponding to the various frequency bands and gives a multi-resolution representation of the image with localization in both spatial and frequency domains.

Images can be modeled using homomorphic systems, in which a logarithmic transformation is used to convert multiplicative superposition to additive superposition of signals. In such systems linear prediction can be compatible [1]. Linear prediction model optimally extract information about a current sample from a neighbourhood of samples in its causal past [2]. The process of signal or system modeling removes redundancy, which is the essence of data compression [3].

DOI : 10.5121/ijma.2012.4105 
The International Journal of Multimedia \& Its Applications (IJMA) Vol.4, No.1, February 2012

In our proposed method the image is decomposed into approximation and details at multi-scale. The highest level approximation and details are retained. In addition to that a few coefficients of details in every level are also retained. Using linear prediction on these coefficients the details are reconstructed. The approximation of a lower level is reconstructed from higher level coefficients using inverse wavelet transform (IWT). The predicted coefficients are used here. Finally the original image is reconstructed in this manner.

\section{THEORY}

A brief revision of theory associated with the proposed method is given below. The areas dealt here are image compression fundamentals, wavelet transform, linear prediction and correlation properties of wavelet.

\subsection{Image Compression Fundamentals}

In most of the images, the neighboring pixels are correlated, and image contains redundant information. By compressing an image we should find a less correlated representation of the image. Image compression relies on reduction of redundancy and irrelevancy. Redundancy reduction removes duplication from image, and irrelevancy reduction omits parts of the signal that will not be noticed by Human Visual System (HVS). The redundancies in an image can be identified as spatial redundancy, coding redundancy and psycho visual redundancy. Image compression aims at reducing the number of bits needed to represent an image by removing the redundancies as much as possible. [4]

Image compression methods can mainly be classified as lossy compression and lossless compression. In loss less compression the decompressed image will be an exact replica of the original image without any loss in data. It offers a compression ratio of around 2 to 4 . Lossy compression will not result in exact recovery of original image. Some fine details are sacrificed to obtain better compression ratio. The compression ratio in this case can exceed 100[5]. Wavelet compression methods are one of the most popular compression methods. Due to symmetric impulse response bior9/7 wavelet is suitable from compression perspective [6].

\subsection{Wavelet Transform}

Discrete dyadic wavelet transforms have been successfully applied to solve different problems in many fields, owing to the good spatial localization and fairly good frequency localization of their bases [7], [8]. They are invertible and efficient. When applied to an image, the image is split into its details at different scales, orientations and positions. The transformed image is de-correlated [9].

One-dimensional wavelet theory defines a function $\psi$, the wavelet, and its associated scaling function $\phi$, such that the family of functions $\left\{\psi^{j}(x)\right\} \mathrm{j} \in Z$, are orthonormal, where

$$
\psi^{j}(x)=\sqrt{2^{j}} \psi\left(2^{j} x\right)
$$

The wavelet transform can be implemented by quadrature mirror filters $G=(g(n))$ and $H=$ $(h(n)), n \in Z$, where

$$
h(n)=1 / 2\langle\phi(x / 2), \phi(x-n)\rangle ; g(n)=(-1)^{n} h(n)
$$

( < >denotes $L^{2}$ inner product). $H$ corresponds to a low-pass filter, and $G$ is an octave wide highpass filter. The reconstruction filters have impulse responses as given in Equation(3) [10]. 
The International Journal of Multimedia \& Its Applications (IJMA) Vol.4, No.1, February 2012

$$
h^{*}(n)=h(l-n) ; g^{*}(n)=g(l-n)
$$

A group of transform coefficients resulting from the same sequence of low pass and high pass filtering operations gives approximation and detail coefficients respectively.

Two Dimensional Discrete Wavelet Transform (2D DWT) is performed by applying a separable filter bank to the image. Applying the one dimensional transform in each row, we get two subbands- approximation and details coefficients in each row ( $L$ and $H$ subbands). Then applying one dimensional DWT column-wise on these $L$ and $H$ subbands, four subbands $L L, L H, H L$, and $H H$ are obtained. $L L$ is a coarser version of the original input signal called approximation image. $L H, H L$, and $H H$ are the high frequency sub-bands containing the detail information (vertical, horizontal and diagonal details images). The number of decompositions performed on original image to obtain sub-bands is called sub-band decomposition [11]. Figure 1 shows 2D DWT performed in separable form on an image and the two level decomposition of the image.

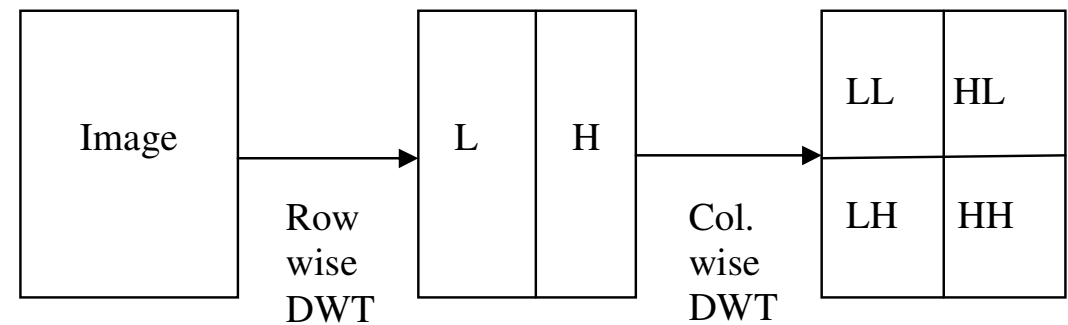

(a)

(b)

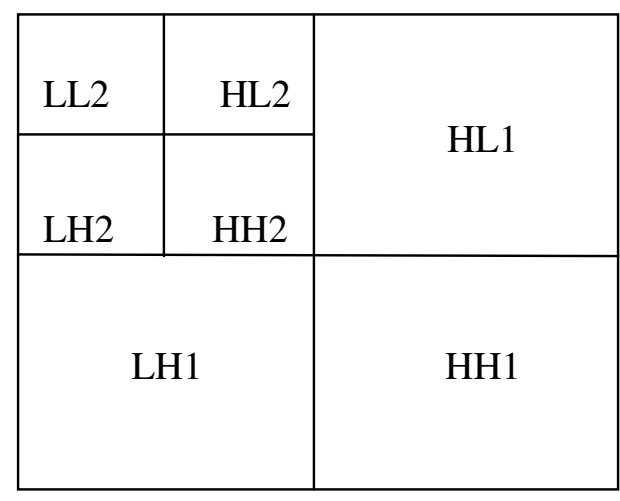

Figure 1. 2D DWT performed in separable form on an image

a) First level decomposition b) Second level decomposition

\subsection{Linear Prediction}

Linear Predictive Coding (LPC) is a popular and efficient technique mainly used in signal compression and speech processing. The signal is modeled as a linear combination of its past values and a hypothetical input to a causal system whose output is the given signal. In the frequency domain, this is equivalent to modeling the signal spectrum by a pole zero model [3]. The sample of the signal is predicted and if the prediction is done from weighted sum of other samples of the signal, the linear predictive model is auto regressive (AR) model [12]. The model parameters are obtained by a least squares analysis in the time domain and frequency domain [3]. The AR model of a process expresses it as finite linear aggregate of its previous values. Let us 
The International Journal of Multimedia \& Its Applications (IJMA) Vol.4, No.1, February 2012

denote the values of stochastic process at equally spaced times, $n,(n-1),(n-2) \ldots$ by $y(n), y(n-1), y(n-$ $2), \ldots \ldots$

$$
y(n)=a_{2} y(n-1)+a_{z} y(n-2)+a_{p} y(n-p)
$$

Exploiting autocorrelation values the Yule-Walker equations can be arrived at.

$$
R_{y y}(k)=\sum_{m=1}^{p} \alpha_{k} R_{y y}(m-k)
$$

Using Levinson- Durbin algorithm [13] the above equations can be solved. Levinson Durbin algorithm is as follows:

1. Initialise the recursion

$$
\begin{aligned}
& \alpha_{0}(0)=1 \\
& \varepsilon_{0}=r_{y y}(0)
\end{aligned}
$$

2. For $\mathrm{k}=0,1, \ldots \mathrm{M}$

$$
\begin{aligned}
& \gamma_{p-1}=r_{y y}(p)+\sum_{k=1}^{p-1} \alpha_{p}(k) r_{y y}(p-k) \\
& \Gamma_{p}=-\frac{\gamma_{p-1}}{\epsilon_{p-1}} \\
& \alpha_{p}(k)=\alpha_{p-1}(k)+\Gamma_{p} \alpha_{p}^{*}(p-k) \\
& \alpha_{p}(k+1)=\Gamma_{p} \\
& \varepsilon_{p}=\varepsilon_{p}\left[1-\left|\Gamma_{p}\right|^{2}\right]
\end{aligned}
$$

Thus we can obtain the predictor coefficients. Using these coefficients, the signal can be predicted using (4).

\subsection{Correlational Properties of Wavelet Coefficients}

Let $f(t)$ be a signal whose WT is calculated. Let $C A_{j}$ and $C D_{j}$ be the approximation and details coefficients at decomposition level $j$. We assume the signal to be stationary and so transform coefficients also should have same property. The expectation of approximation coefficients at the $j^{\text {th }}$ level can be given as 
The International Journal of Multimedia \& Its Applications (IJMA) Vol.4, No.1, February 2012

$$
E\left[C A_{j}\right]=\sqrt{2^{-j}} \int f(t) E_{n}\left[\Phi\left(2^{-j} t-n\right)\right] d t \quad \approx \frac{\sqrt{2^{-j}}}{N} \int f(t)
$$

where En is an average operator while $N$ is the length of support range of $\left[\Phi\left(2^{-j} t-n\right)\right]$. The expectation of approximation coefficients is proportional to the average of the original signal. It can also be found that detail coefficients have zero mean.

$$
E\left[C D_{j}\right]=\sqrt{2^{-j}} \int f(t) E_{n}\left[\Psi\left(2^{-j} t-n\right)\right] d t=0
$$

The autocorrelation of $C A_{j}$ and $C D_{j}$ are proportional to autocorrelation of original signal. $C A j$ and $C D j$ are decorrelated. The detail coefficients at different levels are also decorrelated. The higher the correlation, the more is the scope for redundancy removal in prediction filtering [14].

\section{ThE PROPOSED METHOD}

The proposed method utilizes the correlation of localized WT coefficients. This property will lead to more correlation of wavelet coefficients in the same prediction channel. In this method, One Dimensional (1D) DWT is performed along the rows/columns of the original image up to four levels and fourth level WT coefficients are retained. Predictor coefficients for each row/column in detail coefficients at $(i-1)^{\text {th }}$ level is calculated. Few prediction coefficients and that much detail coefficients, at each level along with retained coefficients from fourth level forms the compressed image.

In the decompression, with a few predictor coefficients and that much detail coefficients in the $i^{\text {th }}$ level, all the details coefficients in the $i^{\text {th }}$ level are reconstructed. Approximation coefficients for $i^{\text {th }}$ level is obtained from inverse DWT on $(i+1)^{\text {th }}$ level of wavelet coefficients. Using approximation and detail coefficients of $i^{\text {th }}$ level, approximation of $(i-1)^{\text {th }}$ level is obtained. Thus the whole image is reconstructed.

The algorithms developed for the proposed method is shown below. This can be applied on the image either along the rows or columns.

\section{Algorithm}

\section{Compression}

\section{Step1: Select the image of interest and input the no. of coefficients, $n$ to be retained.}

\section{Step2: Perform DWT on the image.}

Decompose the image into wavelet transform coefficients applying 1D- DWT on the image row/column wise.

\section{Step3: Retaining the coefficients}

Retain the fourth level decomposition coefficients so that they can be used for reconstruction. In addition to that $n$ rows/columns of each level are also retained.

\section{Step4: Obtain the predictor coefficients.}

Take the first row/column and find $n$ predictors for first row/column of detail coefficients. Similarly find $n$ predictors for first $n$ rows/columns. Also find column-wise/row-wise $n$ predictors for the remaining columns/rows. Do this for all the levels of decomposition. 
The International Journal of Multimedia \& Its Applications (IJMA) Vol.4, No.1, February 2012

\section{Decompression}

\section{Step1: Reconstruction of detail coefficients.}

a) Initialise an array for predicted coefficients.

b) Using first $n$ coefficients in the row/column and $n$ predictors the whole row/column is reconstructed. It is done for $n$ number of rows/columns.

c) Now consider the first column/row. We have $n$ elements in first column/row. Using the $n$ column/row predictors the whole column/row is reconstructed.

d) Repeat step (c) for all columns/rows.

\section{Step2: Reconstruction of approximation coefficients}

Applying 1D-IDWT on the corresponding rows/columns on $(i+1)^{\text {th }}$ level coefficients, $i^{\text {th }}$ level approximation is reconstructed.

\section{Step3: Reconstruction of previous levels.}

Steps 1 and 2 are repeated till $i=$ zero. Thus the original image is reconstructed from prediction.

\section{Evaluation Criteria}

For assessing fidelity of reconstructed image, there are two classes of criteria; objective fidelity criteria and subjective fidelity criteria. In objective fidelity criteria the level of information loss is expressed as a function of input image, compressed image and subsequently decompressed image. Some of the objective fidelity criteria are normalized mean square error (NMSE), normalized absolute error (NAE), and peak signal to nose ratio (PSNR) [11]. The measures like compression ratio (CR) and bit per pixel (bpp) also evaluate a compression method.

Let $x(m, n)$ be the original $\mathrm{M} \times \mathrm{N}$ pixel image and $x^{*}(m, n)$ be the reconstructed image

$$
\begin{gathered}
\text { NMSE }=\frac{\sum_{m=1}^{M} \sum_{n=1}^{N}\left[x(m, n)-x^{*}(m, n)\right]^{2}}{\sum_{m=1}^{M} \sum_{n=1}^{N}[x(m, n)]^{2}} \\
N A E=\frac{\sum_{m=1}^{M} \sum_{n=1}^{N}\left[x(m, n)-x^{*}(m, n)\right]}{\sum_{m=1}^{M} \sum_{n=1}^{N}[x(m, n)]} \\
P S N R=10 \log _{10} \frac{255}{M S E}
\end{gathered}
$$

The subjective fidelity criteria measure the quality of an image by human evaluation using an absolute rating scale. One possible rating scale is as shown below. $\{-3,-2,-1,0,1,2,3\}$ which represent \{much worse, worse, slightly worse, the same, slightly better, better, much better\} [15].

\section{RESULTS AND DISCUSSIONS}

The images with large dimensions i.e., greater than 1024 x 1024 are taken as test images. The test images constitute both medical and natural gray scale images. Biorthogonal wavelets are used in this method. The algorithm is applied row wise and column wise on the images and results are compared. The original and reconstructed images using row-wise decomposition are shown in the Figure 2 and Figure 3 
The International Journal of Multimedia \& Its Applications (IJMA) Vol.4, No.1, February 2012

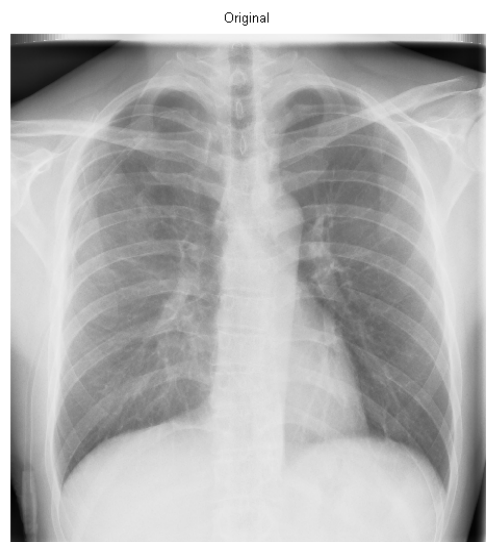

(a)

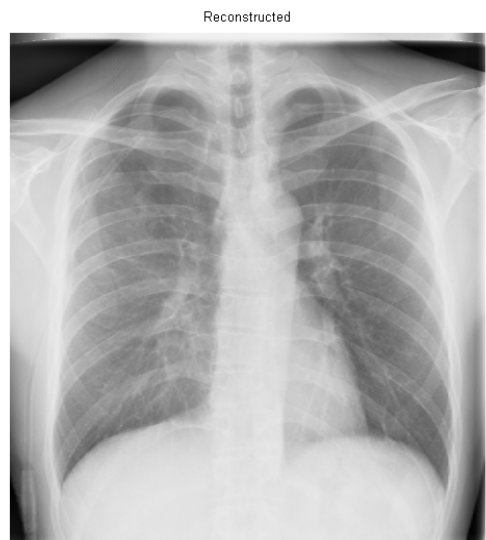

(b)

Figure 2. Decompressed biomedical image at 14:1 using row-wise decomposition a) Original image b) decompressed image

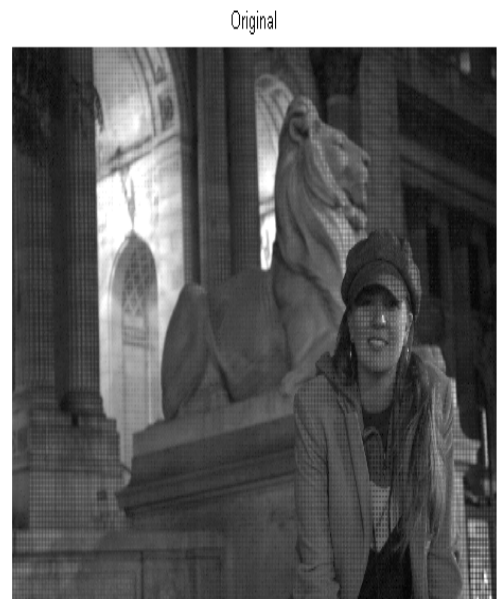

(a)

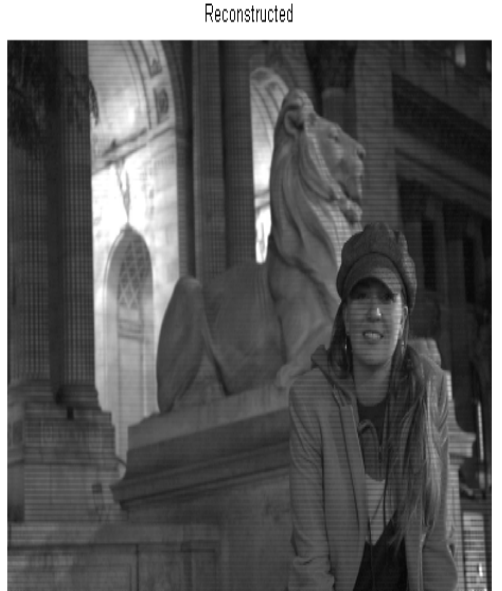

(b)

Figure 3. Decompressed image at 14:1 using row-wise decomposition a) Original image b) decompressed image

The magnified versions of original and decompressed images are shown for comparison in Figure 4 and Figure 5. 
The International Journal of Multimedia \& Its Applications (IJMA) Vol.4, No.1, February 2012

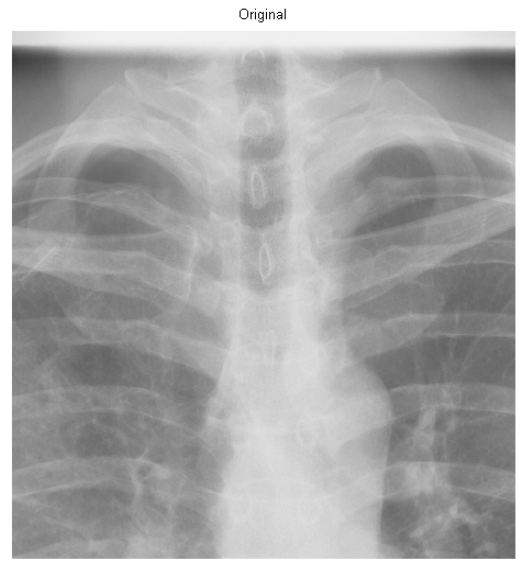

(a)

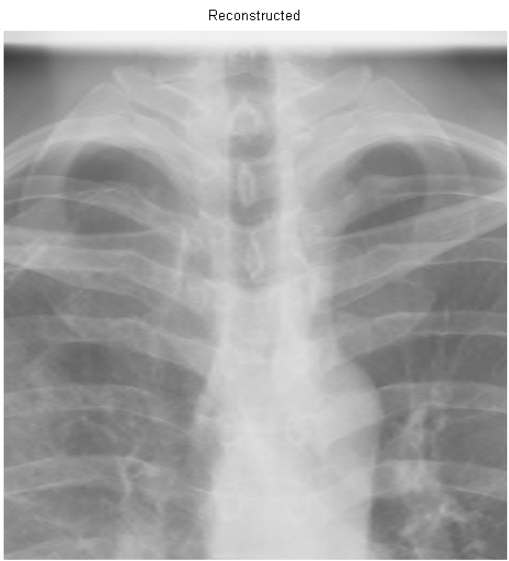

(b)

Figure 4. Biomedical image -zoomed by 2 using row-wise decomposition a) Original image b) decompressed image

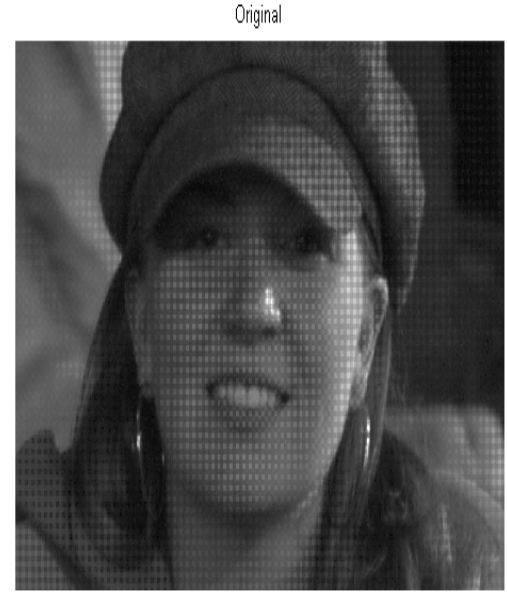

(a)

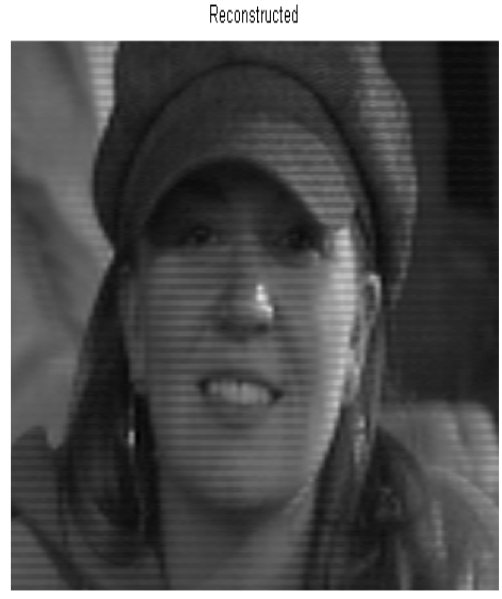

(b)

Figure 5. Real world image -zoomed by 4 using row-wise decomposition a) Original image b) decompressed image

The original and reconstructed images using column-wise decomposition in the algorithm are shown in the Figure 6 and Figure 7. 
The International Journal of Multimedia \& Its Applications (IJMA) Vol.4, No.1, February 2012

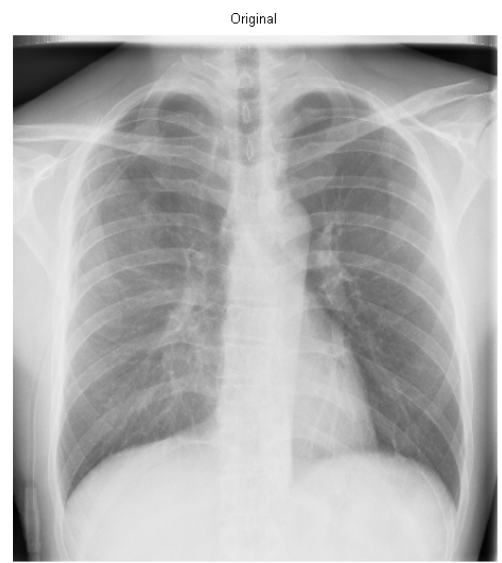

(a)

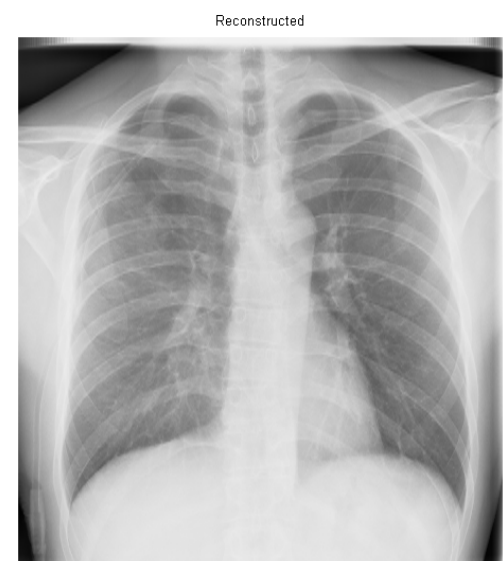

(b)

Figure 6. Decompressed biomedical image at 14:1 using column-wise decomposition a) Original image b) decompressed image

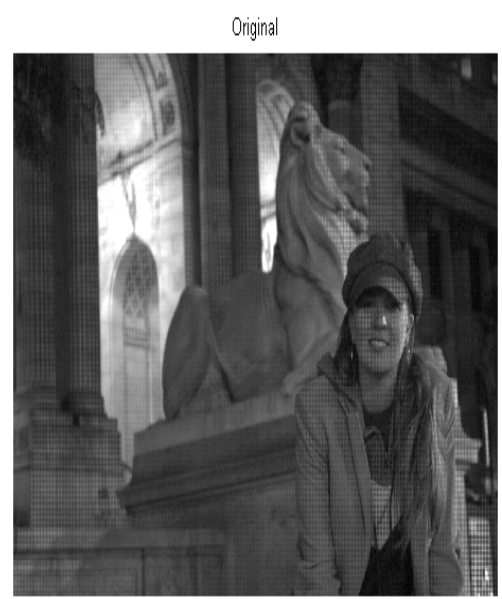

(a)

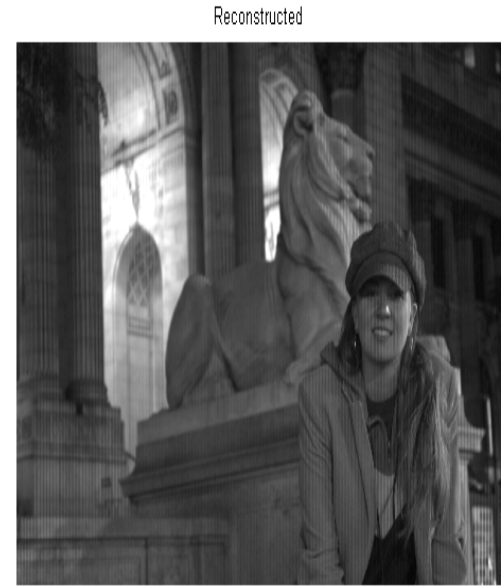

(b)

Figure 7. Decompressed image at 14:1 using column-wise decomposition a) Original image b) decompressed image

The magnified versions of original and decompressed images are shown for comparison in Figure 8 and Figure 9. 
The International Journal of Multimedia \& Its Applications (IJMA) Vol.4, No.1, February 2012

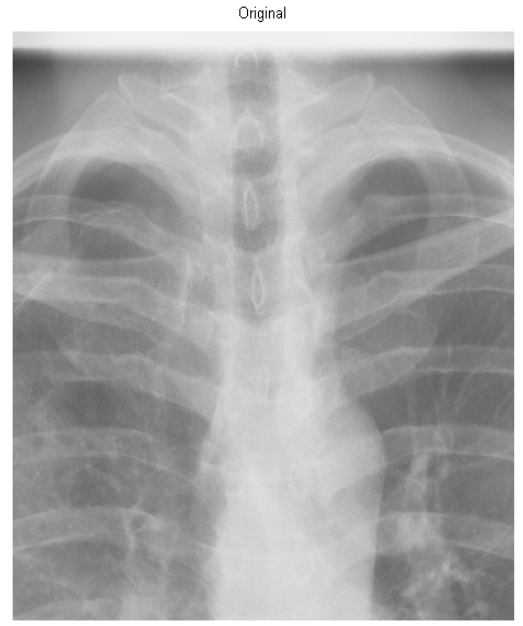

(a)

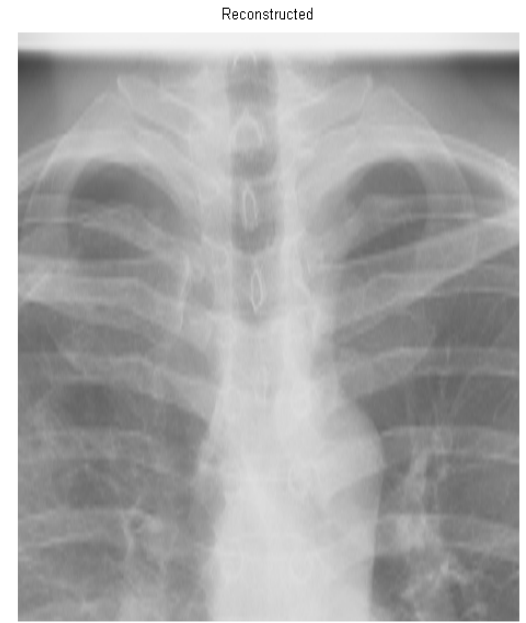

(b)

Figure 8. Biomedical image -zoomed by 2 using column-wise decomposition a) Original image b) decompressed image

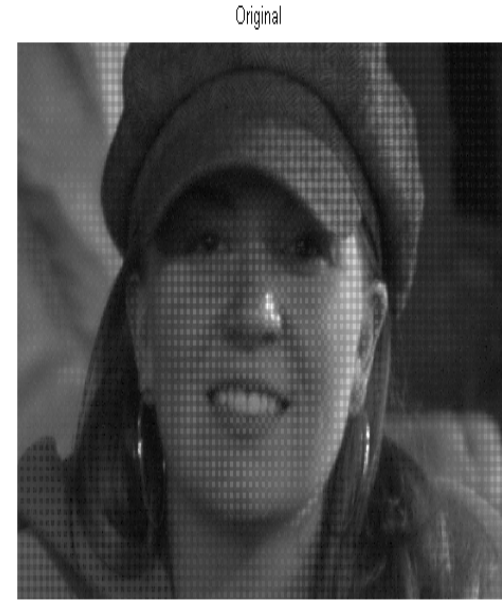

(a)

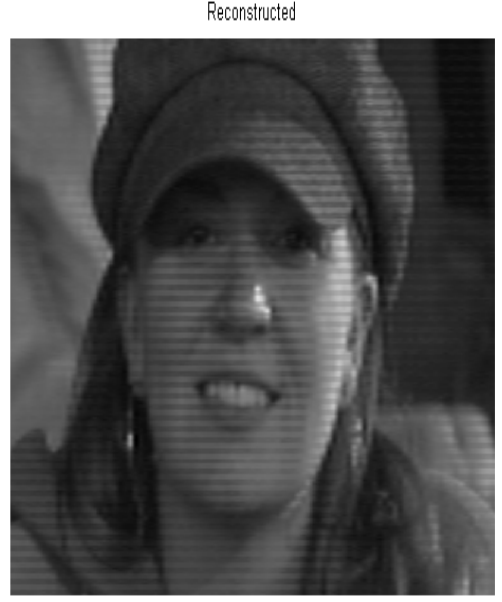

(b)

Figure 9. Real world image -zoomed by 2 using column-wise decomposition a) Original image b) decompressed image

The performance of the algorithm is validated using objective criteria like PSNR, NMSE, NAE and BPP and various performance curves are shown in Figure 10. It is found that as compression ratio increase PSNR first decrease and then increases. The column-wise decomposition gives a better performance as there is not much variation in PSNR values with compression ratio (CR). This method gives as high as $45 \mathrm{~dB}$ for some images. NMSE and NAE is less for column-wise decomposition compared to row-wise decomposition. The variation of CR with bpp is almost same in the case of both the decompositions. 
The International Journal of Multimedia \& Its Applications (IJMA) Vol.4, No.1, February 2012

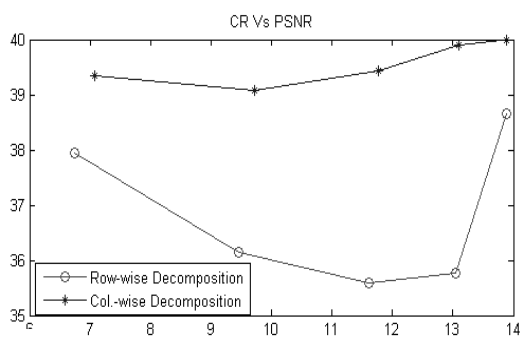

(a)

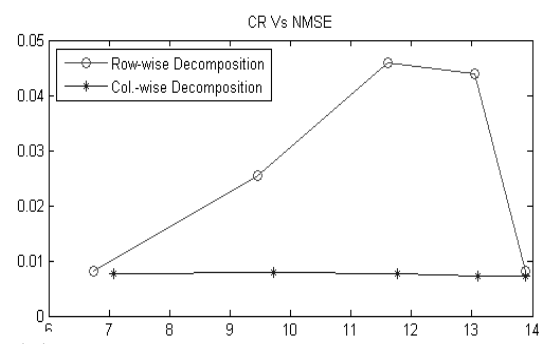

(c)

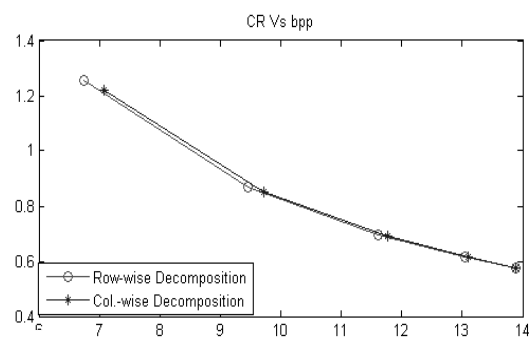

(b)

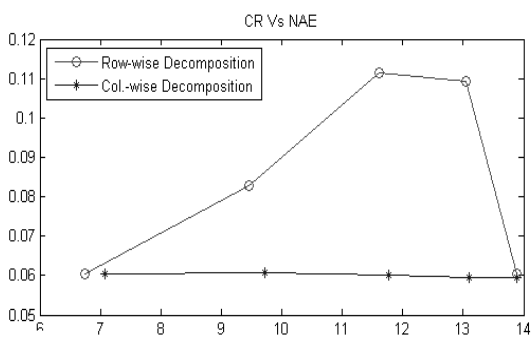

(d)

Figure 10. Performance Curves a) PSNR b) NMSE c) bpp d) NAE

The variation of the parameters PSNR, bpp, NMSE and NAE with no. of coefficients retained is also plotted in Figure 11. The curves are found to be mirror reflection of that with CR.

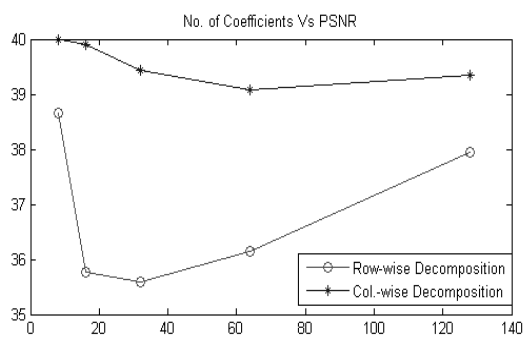

(a)

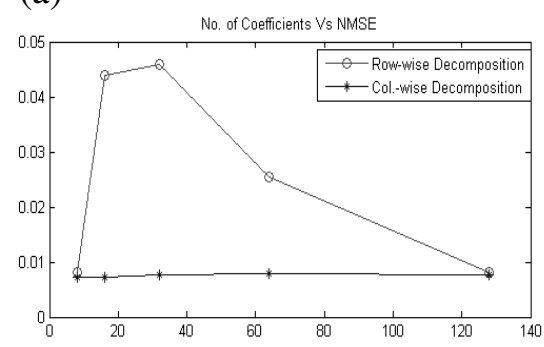

(c)

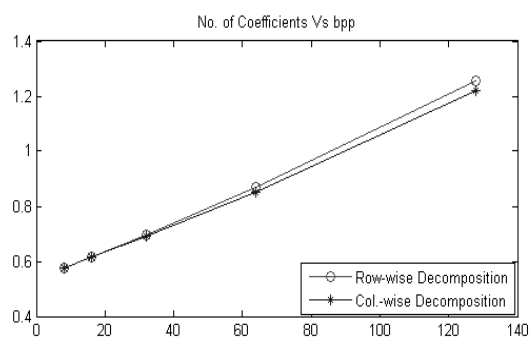

(b)

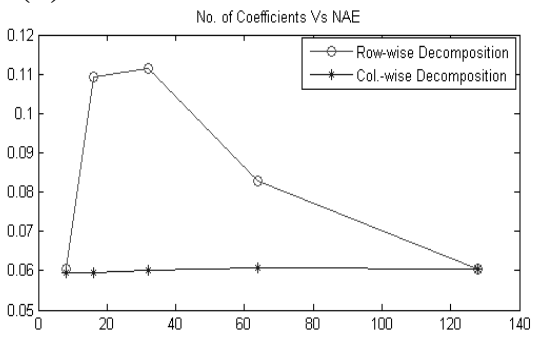

(d)

Figure 11. Performance Curves a) PSNR b) NMSE c) bpp d) NAE

The time taken for compression and decompression is an important parameter to be considered when the image has to be send across a network. The total time taken is plotted against no. of coefficients retained and CR. The plots are shown in Figure 12. The time taken first decreases with increase in no. of coefficients retained and then it start increasing for both the algorithms. The algorithm with column-wise decomposition is found to have consistent performance as the 
variations are not large. The time taken decreases rapidly with $\mathrm{CR}$ at lower values and changes slowly at higher CR.

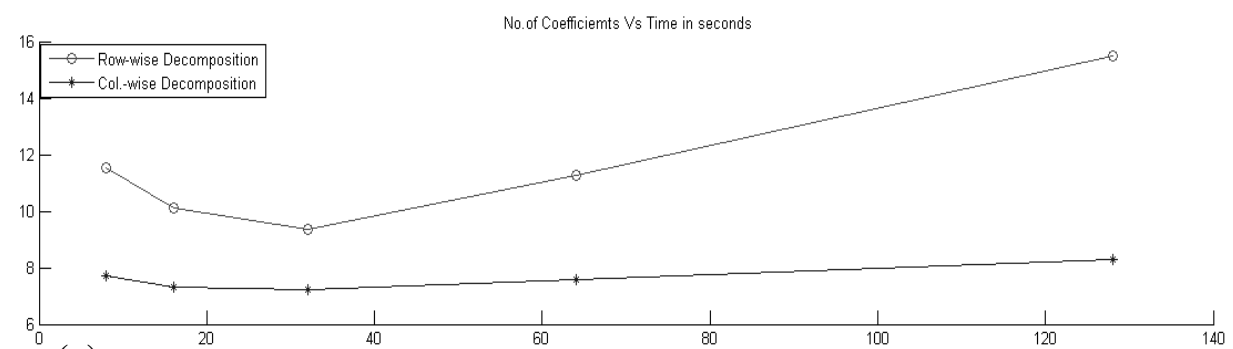

(a)

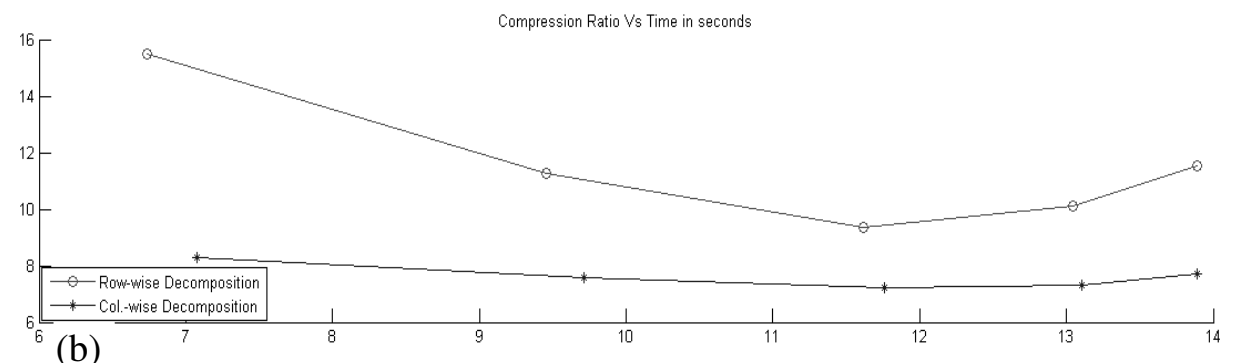

Figure 12. Performance Curve in terms of time taken a) No. of Coefficients b) CR

The algorithm was validated using Model Fest database [16]. This database consists of images that are high quality HVS models that can also be used to improve compression technologies. All the images are of size $256 \times 256$. The images in this database are classified into different categories. They are Gabor patch with fixed size, fixed cycles and an optional category with different phase, elongated Gabor patch, Gabor patch with different aspect ratio, Gabor patch with different orientation, Gabor patch with different sub-threshold summation, collinear patches, plaid, multi-poles, Gaussian blob, white noise and miscellaneous.

Out of the 47 images in the database, algorithm worked fine with 39 images. For multi-poles category image model the algorithm showed best result. For example, for the image Line31, PSNR is as high as $90 \mathrm{~dB}$ while NMSE is almost zero and NAE is very low. Among the categories in the database, the row-wise decomposition is suitable for Gabor patch with fixed size, fixed cycles and an optional category with different phase. The reconstruction was possible with as less as 5 coefficients. The PSNR ranged from 53 to $73 \mathrm{~dB}$. In the Gabor patch with fixed cycles category, the one model with high standard deviation after reconstruction contained noise. The noise can be reduced by increasing the number of coefficients retained. The PSNR can be improved to $55 \mathrm{~dB}$ by compromising on CR while NMSE and NAE also reduced.

The elongated Gabor patch, Gabor patch with different aspect ratio, Gabor patch with different orientation, Gabor patch with different sub-threshold summation categories also showed good results for this method. The elongated Gabor patch and patch with different aspect ratio gives PSNR as high as $73 \mathrm{~dB}$, NMSE 0 and NAE as low as 0.000046 .

For collinear patch category, the reconstructed images are corrupted by noise but still recognisable. Here also increase in number of coefficients retained, removes noise and improves the performance in terms of PSNR, NMSE and NAE. But CR reduces. The improvement achieved in this case is achieved by retaining only 10 coefficients.

The plaid category model can also be compressed with this algorithm without affecting the quality of images. In Gaussian blob category, while algorithm works good for those with higher standard deviation, noise is introduced in the reconstructed versions of those with less standard 
deviation. Even though noise removal is possible with increase in number of retained coefficients, ringing is observed in the output.

In the white noise category we have 2 stimuli-fixed white noise and random white noise. For both the stimuli, the algorithm could not work well. The introduced noise rendered the reconstructed image poor visual quality even when number of coefficients was increased.

In the miscellaneous category, we have Bessel function stimulus which is narrow band in spatial frequency but broad band in orientation. This method gives good quality reconstructed image for Bessel function stimuli image. We also have disk image in this category which is a familiar target used in medical imaging research. With this image, on reconstruction the edges are smoothed out and ringing effect is observed. We also have a natural image whose visibility cannot be predicted by any models. The reconstructed image in this case is a blurred one even though noise can be removed by increasing the number of coefficients. The checker board image is another one in the miscellaneous category. The checker board image have salient properties in the spatial domain are a set of high contrast edges that have no corresponding frequency components [17].

In modelfest database majority of the images have horizontal orientation and algorithm using row-wise decomposition is best suited in this case. Some of the original and reconstructed images from ModelFest database using row-wise decomposition algorithm are given in the Figure 13 and Figure 14.

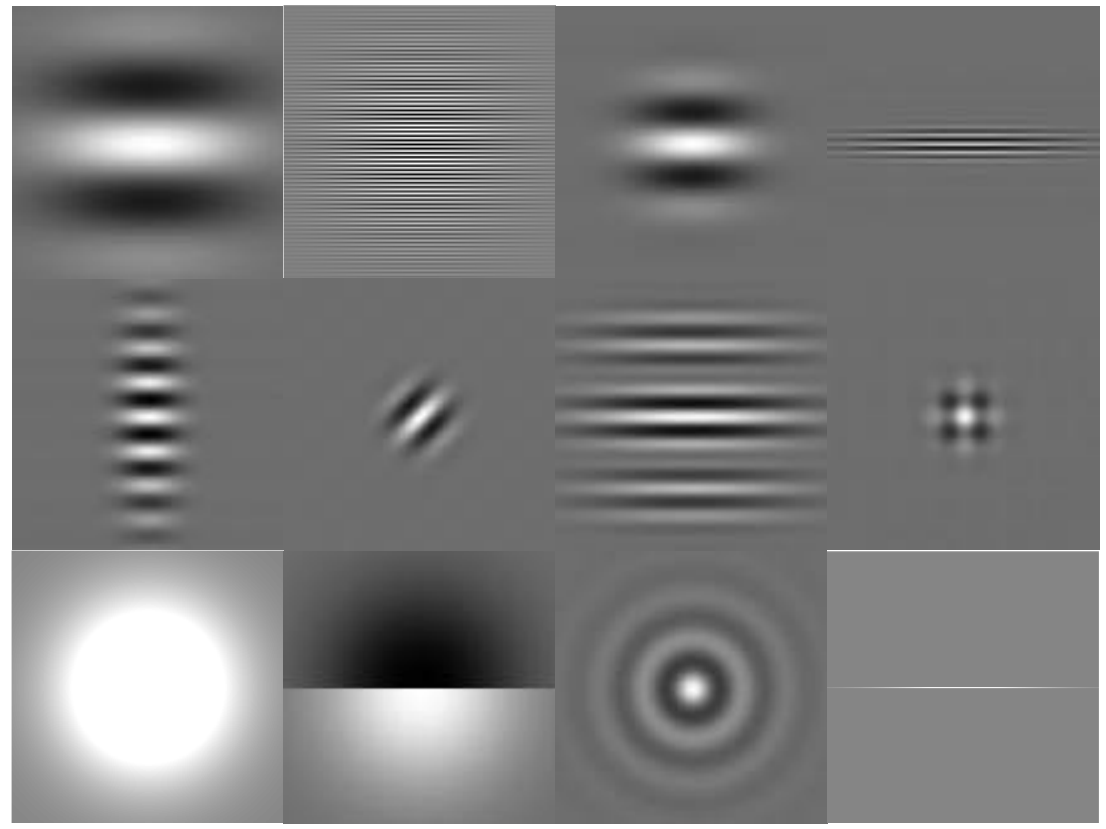

Figure 13. Original Images from Model Fest database 
The International Journal of Multimedia \& Its Applications (IJMA) Vol.4, No.1, February 2012

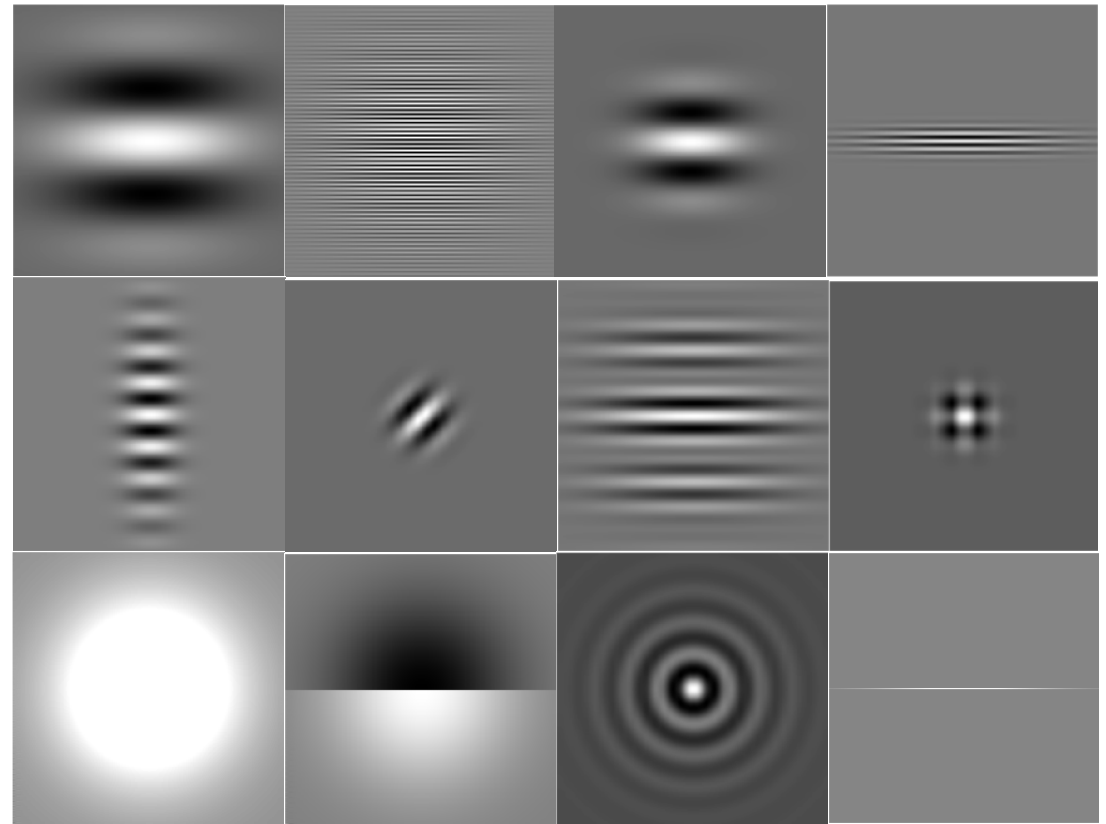

Figure 14. Reconstructed Images Using row-wise decomposition

On all the images, the algorithm using column-wise decomposition is also tried out. The algorithm worked well for many images namely Gabor patch with different phase category. The algorithm using column-wise decomposition when applied to those images after giving a rotation of ninety degrees is found to give good results. The images Bessel, Gaussian blob with higher standard deviation, Gabor patch with different orientation and plaids showed good results even without rotation of the images. The rotated images and their reconstruction using columnwise decomposition are shown in Figure 15 and Figure 16.

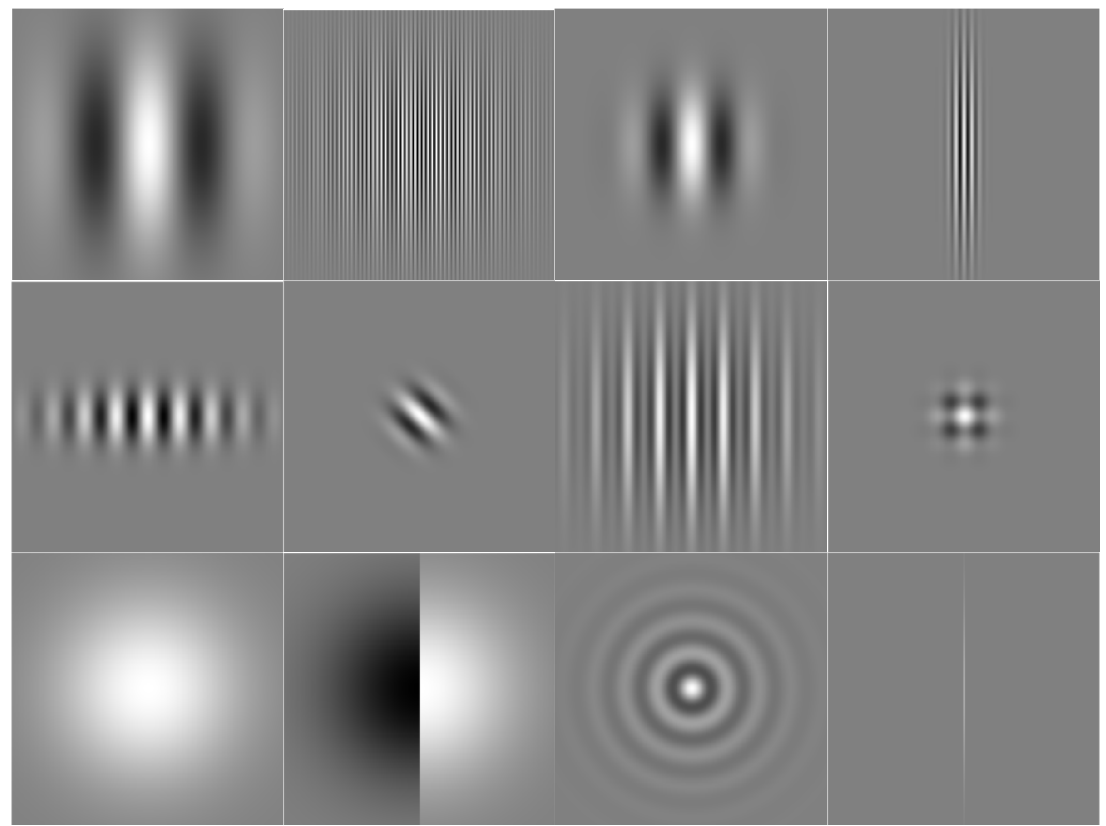

Figure 15. Original Images from Model Fest database rotated by 90 degrees 
The International Journal of Multimedia \& Its Applications (IJMA) Vol.4, No.1, February 2012

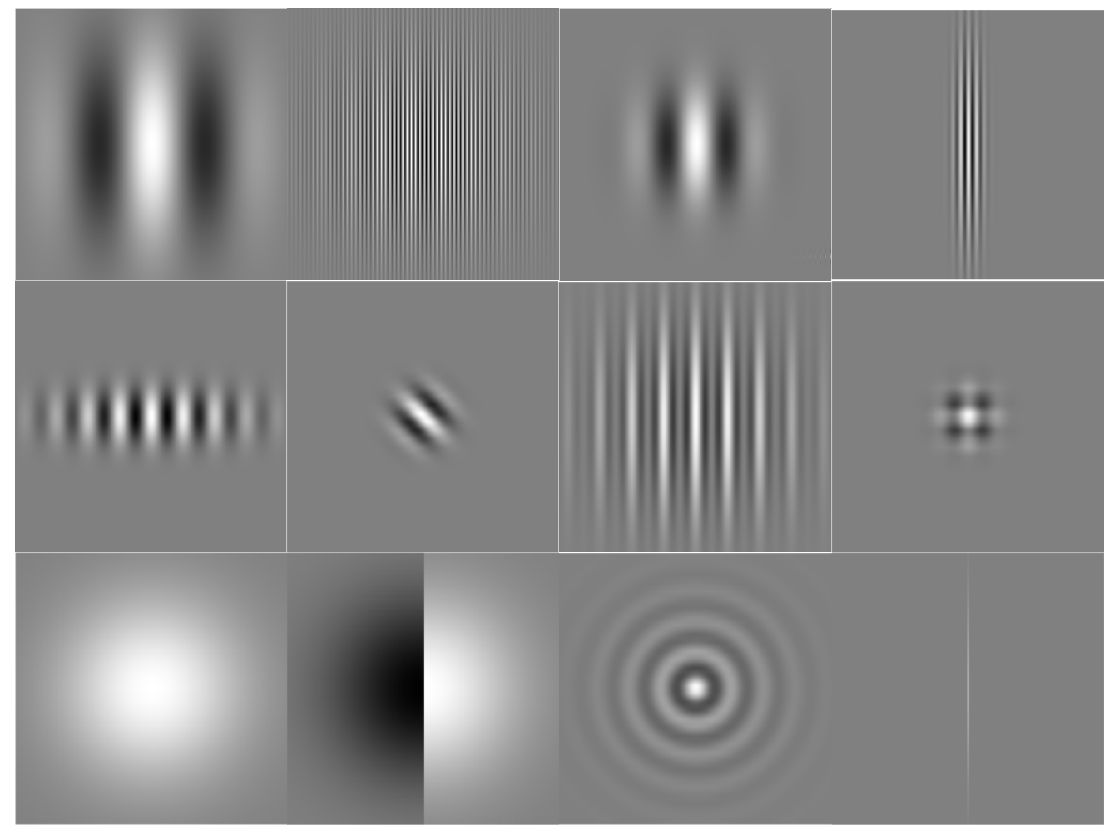

Figure 16. Reconstructed Images Using column-wise decomposition

The subjective assessment of the reconstructed images is also done. The original and decompressed images were shown to different viewers and there were asked to rate the images. As per the feedback this method is found to be effective in compressing the images without losing their vital information.

\section{CONCLUSIONS}

The proposed method works better with images of large dimensions. This method gives same compression ratio and bpp on images of same size. Here the correlation of each row/column in the image with its wavelet coefficients exploited to predict the next wavelet coefficients. The prediction can be done with number of coefficients as small as five. The performance method is evaluated on the basis of subjective criteria and parameters like PSNR, NAE, NMSE and bpp. The experimental results show that the proposed method, in addition to achieving good compression gives a better performance. We can conclude that for images with horizontal orientation 1D-DWT taken row wise gives a better result while taking 1D-DWT column wise suits images with vertical orientation.

\section{REFERENCES}

[1] Maragos, P., Schafer, R.W \& Mersereau, R.M (1984) "Two-Dimensional Linear Prediction and Its Application to Adaptive Predictive Coding of Images processing in the context of a visual model", IEEE Transactions on Acoustics, Speech and Signal Processing, Vol. ASSP 32, No. 6, pp1213-1229.

[2] Rajagopalan, R., Orchard, M.T., Ramchandran, K (1996) "Optimal Supports for Linear Predictive Models”, IEEE Transactions on Signal Processing, Vol. 44, No. 12, pp3150-3153.

[3] Makhoul, J, (1975) "Linear Prediction: A tutorial Review", Proceedings of IEEE, Vol. 63, No. 4, pp561-579.

[4] Kharate, G.K., Patil, V.H. \& Bhale, N.L, (2007) "Selection of Mother Wavelet for Image Compression on Basis of Nature of Image", Journal of Multimedia, Vol. 2, No. 6, pp44-51. 
The International Journal of Multimedia \& Its Applications (IJMA) Vol.4, No.1, February 2012

[5] Vidhya, K. \& Shenbagadevi, S, (2009) "Performance Analysis of Medical Image Compression", Proceedings of IEEE International Conference on Signal Processing Systems, pp979-983.

[6] Masud, S. \& Canny, J.V.M, (1998) "Finding a Suitable Wavelet for Image Compression Applications", Proceedings of IEEE International Conference on Acoustics, Speech and Signal Processing, Vol. 5, pp2581-2584.

[7] Strang, G \& Nguyen, T (1996) Wavelets and Filter Banks, Wellesley Cambridge Press.

[8] Mallat, S (1999) A Wavelet Tour of Signal Processing, Academic Press.

[9] Ruedin, A.M.C. \& Acevedo, D.G, (2005) "Prediction of coefficients for Lossless Compression of Multispectral Images", Proceedings of Satellite Data Compression, Communications, and Archiving, SPIE, Vol. 5889, pp202-215.

[10] Lewis, A.S. \& Knowles, G, (1992) "Image Compression Using the 2-D Wavelet Transform", IEEE Transactions on Image Processing, Vol. 1, No. 2, pp244-250.

[11] Shahhoseini, E., Nejad, N.A., Behnam, H. \& Shahhoseini, A (2010) “A new approach to compression of medical ultrasound images using wavelet transform”, Proceedings of IEEE International Conference on Advances in Circuit, Electronics and Micro-electronics, pp40-44.

[12] Hu, J.H., Wang, Y. \& Cahill, P.T, (1997) "Multispectral Code Excited Linear Prediction Coding and Its Application in Magnetic Resonance Images", IEEE Transactions on Image Processing, Vol. 6, No. 11, pp1555-1566.

[13] Hayes, M.H (1996) Statistical Digital Signal Processing and Modeling, John Wiley.

[14] Huang, J.S., Nguyen, D.T., Negnevitsky, M. \& Philips, C.J.E, (1999) "Correlation Properties of Wavelet Transform and Applications in Image Coding", Proceedings of IEEE International Symposium on Signal Processing and its Applications, pp611-614.

[15] Gonzalez, R.C. \& Woods, R.E (2006) Digital Image Processing, Prentice-Hall. India.

[16] Carney, T.: ModelFest Web Site-

http://www.neurometrics.com/projects/Modelfest/IndexModelfest.html

[17] Carney, T., Klein, S.A., Tyler, C. W., Silverstein, A. D., Beutter, B., Levi, D., Watson, A. B., Reeves, A. J., Norcia, A.M., Chen, C.-C., Makous, W. \& Eckstein, M. P( 1999) "The development of an image/threshold database for designing and testing human vision models", Proceedings of SPIE Human Vision, Visual Processing, and Digital Display IX, Vol. 3644, No. 1, pp542-551.

\section{Authors}

Arya Devi P S is a Research Scholar at Model Engineering College, Kochi, Kerala. She obtained her B.E Degree Electronics \& Communication Engineering from Bharatidasan University, received M.Tech in Applied Electronics from Mahatma Gandhi University, Kerala in 2008. Her areas of interest include Image Compression, Information Theory and Coding

M G Mini is Associate Professor and Head of the Department, Electronics, at Model Engineering College, Kochi, Kerala. She has received $\mathrm{PhD}$ from CUSAT, Kerala in 2005. Her areas of interest include Digital Signal and Image Processing, Digital System Design, VLSI\& ASIC Design. She has several research papers published in National and International journals to her credit.
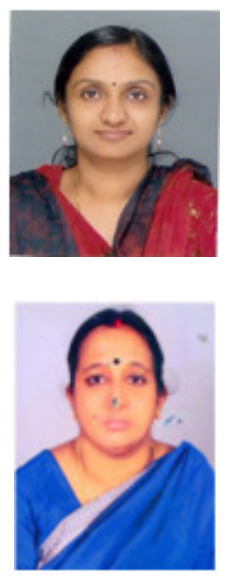